\title{
La creación literaria: una simbiosis trazada por posibilidades y sombras*
}

\author{
César Acosta Narváez ${ }^{1}$ \\ Universidad de Cartagena
}

\section{Resumen}

El siguiente ensayo realiza una aproximación al proceso de creación literaria desde la experiencia de escritura de una novela de ciencia ficción. Reflexiona sobre los diferentes factores y cuestionamientos que circundan al creador literario cuando construye su universo ficcional. Presenta, así mismo, una visión panorámica del género de ciencia ficción en Colombia: un género que ha sido desarrollado desde la periferia.

Palabras clave: creación literaria, ciencia ficción, creador literario, novela, periferia.

\begin{abstract}
Creative writing: a symbiosis stipulated by probabilities and shadows. The following essay realizes an approximation to the process of creative writing from the experience of writing of a novel of science fiction. It reflects about the different factors and questions that surround the literary creator when he constructs his fictional world. The text presents a panoramic vision of science fiction's genre in Colombia: a kind that has been developed from the periphery.
\end{abstract}

Key words: literary creation, science fiction, literary creator, novel, periphery.

\footnotetext{
* Creative writing: a symbiosis stipulated by probabilities and shadows. Recibido: Septiembre, 2012 - Aprobado: Octubre, 2012.

${ }^{1}$ Estudios de Lingüística y Literatura en la Universidad de Cartagena. Miembro del Grupo de Estudio CARIBELE, especializado en enseñanza del español como lengua extranjera. Docente de español en el Centro Catalina (Spanish School). e-mail: jupiter3220@hotmail.com
} 
"Para escribir, no escribo, o apenas no escribo, me contento con forjar proyectos, crear escenas, soñar con situaciones deslavazadas, que me arrastran y donde me sumerjo. ¡Qué extraña es mi cabeza!”.

Gustave Flaubert.

"Por la creación literaria y a través de ella, el hombre se descubre como criatura que participa en una segunda vida".

Albert Béguin.

\section{La construcción de un mundo}

Emprender un proyecto de creación literaria puede considerarse como un proceso sintético, en la medida en que se requiere de la aplicación, la congruencia y la constante revisión de todo un conjunto de conocimientos asociados, por medio de la configuración de un relato y la constitución de personajes determinados en una historia. La interacción entre estos diversos factores puede verse como una simbiosis de elementos extra e intra-textuales, que terminan con lo que sería la figura del bien o mal logrado "homúnculo literario"z: una creación humana motivada y resultante de los intereses particulares de cada escritor.

Si hacemos una reflexión sobre el hecho de crear un texto literario, podremos encontrar entre sus primeras vertientes la incógnita sobre su propósito, un por qué, una explicación, o por lo menos, una posible solución frente al ideal de la escritura, entendida esta, además de un proceso investigativo, como la búsqueda de una finalidad humana. $\mathrm{Y}$ es que de entre todas las construcciones y posibles respuestas sobre la justificación al momento de escribir, podría comenzarse comprendiendo el acto creativo como una habilidad innata en la mente de todos los seres humanos, puesto que -basándonos en la observación de los elementos que componen los espacios cotidianos, y dadas nuestras necesidades individuales o colectivas- inventamos

\footnotetext{
${ }^{2}$ El término "homúnculo" (del latín homunculus, "hombrecillo") se asocia al campo de la ya olvidada alquimia: un ser humano creado artificialmente por el alquimista, luego de un meticuloso proceso de incubación. En este caso, se recurre a la metáfora (de tipo retórica): el homúnculo vendría siendo una representación del texto literario, por ser el producto de una creación netamente humana. La connotación de artificial aplica, en este caso, a los trabajos de creación literaria contemporáneos, en el sentido de que son acompañados por un carácter investigativo, y que se contraponen con las ideas románticas (por decirlo de un modo, "naturales") de que un texto literario es el producto solamente de la experiencia y sensibilidad humana. Para un estudio más amplio, véase Melián (1964).
} 
o modificamos los objetos que nos brindan una mejor calidad de vida, física o psicológica. La imaginación creativa es, pues, una capacidad inherente, que se proyecta en múltiples ejes: uno de ellos es la creación literaria.

La creatividad, siguiendo a Guillermo Sánchez Medina (2003), "es la capacidad de integrar a partir de una forma existente; es el acto por el cual se unen dos elementos. Como consecuencia se origina otro elemento nuevo y distinto que antes no era. Por lo tanto, la creatividad implica hacer, nacer, dar vida, componer y dar origen" (19). De ahí que el escritor amolde la realidad y una los elementos que más le conciernan, según su visión de mundo, y construya una estética del lenguaje. Debemos entender, por otra parte, que esa capacidad creativa es experiencial, y que, en consecuencia, el origen de un texto literario obedece a una diversidad de condiciones objetivas, oníricas, sociales, epistémicas e ideológicas, hasta el punto de que esos agentes inciden en el tipo de discurso o género literario al cual pertenecerá la obra.

No sólo escribimos con el fin de trasmitir un conjunto de ideales o axiomas situacionales, sino que también lo hacemos en concordancia con una dualidad entre la liberación individual ${ }^{3}$ y una intención o propuesta estética. La primera proposición trata la escritura como el reflejo de todas las cargas y demonios internos que circundan en nuestra mente; es un medio que sirve para callar todas las voces que susurran en la imaginación al artista y que originan en él la iniciativa por construir un mundo con las palabras. Desde esta primera instancia, la subjetividad puede convertirse en un factor dominante en la creación, y así mismo, le permite al escritor plantear una percepción particular de la realidad en el texto. Es decir, el artista construye unas determinadas situaciones, imaginarios y discursos a partir de sus propias motivaciones. "El poeta puede tomar este papel, y el 'Yo', desde la locura, será su expresión patológica” (Bravo, 1996: 22). La creación literaria corresponde, en este caso, al producto de un proceso nutrido mayoritariamente por un carácter subjetivo.

\footnotetext{
${ }^{3}$ La liberación podría asociarse con Sigmund Freud (1935) bajo la motivación o el impulso en el comportamiento humano, correspondiente a la ejecución de unas estructuras psicológicas que actúan ante unas necesidades mayoritariamente físicas, que sería lo que él denomina "libido". En la creación literaria el individuo se ve en la necesidad de construir una historia, porque su psique así se lo demanda. Ante ese evento, la mejor forma de liberarse de esa carga es cediendo ante ella, y en su efecto, surge lo que después sería el texto literario. Pero esto no indica que la creación literaria esté sobredeterminada bajo una presunción psicológica; más bien puede decirse que la motivación es uno de los tantos elementos que permiten el desarrollo de la obra.
} 
Nace de la mente y de las experiencias del escritor, que construye su obra así como un pequeño dios estructura meticulosamente su propio universo ${ }^{4}$. No obstante, segmentar el proceso de creación literaria únicamente con las percepciones individuales es una noción excluyente, puesto que una obra no se desarrolla totalmente desde un mero impulso o estímulo, sino a partir de una variedad de senderos entre el creador, la realidad y el texto. Desde este primer punto, por lo tanto, la subjetividad no sería el factor determinante en la construcción del mundo ficcional, pero sí una de sus bases.

La segunda premisa considera la escritura asociada a lo que se denomina la propuesta estética. En dicha proposición, el creador literario trabaja con determinados elementos textuales, llámense "historia", "motivos", "referentes epistémicos", "tiempo", "discurso de personajes" y "atmósferas espaciales", y los configura de tal manera que en su obra devela un sello muy particular. La obra es propia u original al tener unas coordenadas espacio-temporales únicas e irrepetibles en comparación con otros textos, sumado al orden de los acontecimientos durante la narración. El texto literario es entonces, además de una creación, "un campo dinámico, en lugar de un bloque estático de palabras. Este campo cuenta con un determinado rango de referencia, un sistema de tentáculos parcialmente potencial y parcialmente real: hacia el autor, hacia el lector, hacia una situación histórica, hacia el pasado, hacia el presente" (Said, 2004: 215). Con la propuesta estética se construye un lenguaje, una "enunciación" que da cuenta de múltiples corrientes y factores textuales, entrelazados por el artista, que a su juicio construye una peculiar red de significaciones.

\section{El escritor en la investigación: el texto y sus redes}

En el campo de las ciencias sociales y humanas un conocimiento se construye no sólo desde una única disciplina, rama o ciencia, puesto que hay estudios que requieren del aporte de varias fuentes para su desarrollo. A esto se le llama "interdisciplinariedad". De hecho, también es posible mirar la creación literaria bajo una postura interdisciplinar, en el sentido de que no seaúnicamente el resultado de una construcción "espontánea" de un sujeto creador que, a través del

\footnotetext{
4 "El hombre sacude su yugo, se rebela contra la naturaleza como antaño se rebelara Lucifer contra Dios, a pesar que esta rebelión sólo es aparente, pues el hombre nunca estuvo más cerca de la naturaleza que ahora que ya no busca imitarla en sus apariencias, sino hacer lo mismo que ella, imitándola en el plano de sus leyes constructivas, en la realización de un todo en el mecanismo de la producción de nuevas formas". Huidobro \& Lavarrete (1989: 299. las cursivas son nuestras).
} 
uso de un lenguaje literario $^{5}$, fabrica uno o varios mundos ficcionales, sino que debe entenderse como todo un procedimiento investigativo en el que el investigador construye una historia, al mismo tiempo que selecciona y maneja determinadas informaciones sobre temáticas especificas, como parte de un proceso de complementación. Aleyda Gutiérrez (2008) afirma que "en el proceso de escritura hay un problema por resolver, una intención estética que necesita una forma, un deber con la palabra que lleva a la indagación constante: hay en la creación mucho de investigación" (76).

Así como el investigador tradicional parte de la puesta de sentido de una incógnita o problema a resolver, cuya respuesta se va consolidando a lo largo de la tesis, el escritor inicia su trabajo desde una motivación, una visión particular que implica la construcción de una historia en su proceso, de las conexiones con ciertos temas (epistémicos, sociales, históricos etc.) que tratará de forma explícita o no en su discurso. En esta última modalidad, el investigador-creador realiza un proceso, en el que, además de conocer y reflexionar sobre los temas y teorías de un género literario, toma conciencia de la trayectoria literaria e histórica de éste, al tiempo que plantea su propuesta estética. El artista debe: "indagar las leyes generales para cada tipo [...] y las leyes especiales [...]", pues el escritor considera su trabajo "como un problema que puede resolverse, en parte, por las leyes generales y preestablecidas, $\mathrm{y}$, en parte, por leyes especiales que él debe descubrir y acatar" (Casares, 1965: 5). Desde este punto de vista puede decirse que la creación de un texto no es únicamente una creación estipulada, o producto de la susodicha y romántica inspiración que llega al sujeto, sino que también se relaciona con unos elementos extrínsecos e intrínsecos a ella. En otras palabras, crear un texto sabiendo sus características, directrices, límites, contenidos, género, tradición e historia literaria.

\section{El compromiso del artista: las huellas del pasado}

En el trabajo de creación literaria se presenta una reflexión bastante particular sobre el texto literario en sí, sobre las características inherentes a su composición, especialmente una simbiosis entre

\footnotetext{
${ }^{5}$ Por "lenguaje literario" se entiende aquí la construcción de un sistema de expresión que difiere del uso estándar de la lengua. Es una estructura que vela por la mesurable interacción de cada una de sus partes, que van desde los componentes de una simple oración hasta un texto extenso, y que genera en el lector una serie de emociones y reflexiones de la vida en general, tomadas desde la narración o los acontecimientos de la historia de un mundo ficcional. Para una profundización sobre ello, consúltese Carreter (1980).
} 
dos objetivos fundamentales, porque la creación de una obra alude indirectamente a un objetivo o funciones del escritor para con la sociedad. En primer lugar, habría un objetivo humanístico en la creación literaria, y es la interacción entre el artista y el mundo. Un escritor no es solamente un individuo que explora la imaginación y la sensibilidad durante su existencia particular, sino que, además, entiende que su trabajo es el diálogo con unos imaginarios y arquetipos universales, que forman parte de experiencias y pensamientos colectivos, tales como la muerte, la libertad, el poder, la razón y la justicia, por mencionar algunos casos. Estos tópicos han sido dejados en evidencia por la literatura a través de incontables historias, efectuándose por medio de una tradición literaria en un determinado contexto. El escritor debe entender que su conexión con el texto implica indirectamente una función arquetípica ${ }^{6}$, puesto que la literatura ha girado siempre bajo determinadas constantes, independientemente de la época y el espacio en el que se cree una obra.

El segundo objetivo en el escritor es entender el texto narrativo o poético como un aporte al conocimiento, al arte y la investigación dentro del contexto literario. Entendemos que una obra es, ante todo, una puesta de sentido: comprende una visión particular de la realidad con sus propios fenómenos y características, en la cual se desarrollan ciertos hechos. Comprende también que, al terminarse, pierde los vínculos con el autor (la obra adquiere un estatuto independiente y se presenta como una "enunciación" dentro de un contexto histórico). La novela, en este caso, termina siendo parte de una sucesión de obras ya creadas, pues forma un continuidad temporal. Por eso el escritor, además de construir un mundo ficcional, considerado para algunos como novedoso, está cumpliendo una "función histórica". El texto, independientemente de que forme o no

\footnotetext{
${ }^{6}$ La "función arquetípica" en el escritor alude a los planteamientos psicológicos de Jung. Para Jung, así como hay una herencia biológica en los seres humanos, también existe una de tipo psicológica, que pone en evidencia los conceptos mentales de pueblos y culturas al momento de representar la realidad. Un ejemplo son las comunidades con sus respectivas culturas, que independientemente de su lejanía, o desconocimiento absoluto de otras, presentan patrones similares de representación al momento de aludir a elementos sociales y religiosos, como las mitologías. Las imágenes arquetípicas son representaciones de fenómenos mentales que carecen de forma, pero que se proyectan en multitudes de sistemas de signos (Fadiman \& Robert, 1976: 68). En el caso del escritor, hay una transposición de esos contenidos mentales que ya han trabajado escritores en sus textos, y que corresponden a unos ideales universales que develan el pensamiento humano. Esta función puede ser consciente o inconsciente, dependiendo del creador literario.
} 
parte de un canon literario, se convierte en una propuesta para con los lectores de un tiempo y un espacio.

Una de las labores implícitas del escritor en su contexto es mantener viva la memoria de un entorno, trabajar con los imaginarios, ideologías o hechos concretos de una comunidad o comunidades, y utilizarlos como herramientas para moldear la historia o contenidos en su obra. Si hablamos de una función histórica, el escritor es visto como un investigador del pasado, o un sujeto histórico con su genotexto ${ }^{7}$. Realiza indagaciones concretas, estados del arte situacionales, y sobre todo literarios, para consolidar y reforzar los límites de su creación. El investigador-creador es consciente de una tradición literaria que, según Popovic (citado por Laverde, 2006), "ha sido utilizada para designar siempre el pasado literario [...] el tiempo histórico siempre lo realizamos en el presente y surge como la 'síntesis' del pasado y del presente con proyecciones al futuro [...] al punto de que los autores contemporáneos se sumergen en un proceso en el que participa el pasado literario, las normas de la creación y las normas de la recepción" (8). De allí que, para proponer un estilo, una estética del lenguaje o un texto de determinada índole, se deba conocer lo hecho previamente en cuestión del género a tratar. Se presenta entonces una reflexión y crítica sobre el género a nivel diacrónico, pero desde el texto: cómo este, en su composición, responde ante una tradición y toma, rechaza, refuerza o propone tópicos del plano estético.

\section{La retrospectiva: los andamios particulares de la creación}

Suele asociarse la creatividad a la infancia, periodo en el que la imaginación contribuye al desarrollo de la cognición humana. Desde la niñez, la sensibilidad va abarcando múltiples campos, pues el individuo, a partir de la lectura, reflexiona sobre el mundo y sus propias experiencias personales. Después, en la adolescencia y madurez, la creatividad e imaginación influyen tanto en las personas, que incluso pueden verse reflejadas en la comprensión y creación de textos literarios. Tal concepción remite a una "trayectoria literaria" en el creador y puede justificar su trabajo como artista. Se nos presenta entonces un planteamiento válido, si el fin es hablar de un

\footnotetext{
7 “El genotexto programa para la producción, pero es producto de las estructuras de la sociedad y por eso es necesario relacionarlo con ellas [...] está constituido por las condiciones históricas del producto más las condiciones culturales de la sociedad. Inscrita en el momento histórico, esta combinación dinámica de elementos que es el genotexto, programa todo el devenir del texto" (Amoretti, 1992: 58).
} 
proceso de formación, pero no aplicable en todos los casos, ya que la experiencia es, ante todo, un hecho individual, y las relaciones entre los creadores con el mundo difieren las unas de las otras.

En ese sentido, la creación de una novela de ciencia ficción como Némesis, que se ha convertido en nuestra propuesta particular (un fragmento del primer capítulo puede verse en las páginas 203205 de este número de Visitas al Patio), devela un proceso creativo posterior a la infancia. Dicha singularidad no indica la ausencia de factores externos en la iniciativa por inventar un mundo. Es precisamente en la niñez cuando se consolidan factores extraliterarios que no inciden directamente en la creación de textos, pero que, poco a poco, van formando un proyecto personal, hasta el punto de convertirse en una historia. Cada mundo incita la posibilidad de construir una historia con algunos de sus elementos. El formato puede considerarse un andamio, modelo cuyas características estimulan la configuración de otros universos. Dentro del proceso de creación literaria, las influencias pueden variar y abarcar múltiples formatos, como lo es un determinado relato o el capítulo entero de una serie televisiva u obra literaria. Algunos comics de Marvel, mangas, kartoons americanos, animes japoneses y particularmente las series, textos y películas de fantasía o ciencia ficción de los años noventa, hasta la actualidad, han sido muy influyentes en Némesis, pues en ellos se desarrolla un carácter fantástico bastante marcado.

Resulta llamativa la idea de Genette (1993), al decir que el "poeticante" (o creador literario) inventa su propio mundo con base en algunos fundamentos de la realidad. Hay aquí un proceso de imitación ${ }^{8}$ y proyección, pues las directrices y leyes de un relato se relacionan directamente con nuestra materialidad. Ahora bien, las características de esos mundos son clasificadas como géneros o subgéneros, a los cuales se les puede denominar: "tragedia", "arte romántico", "menipea”, "autobiografía”, o de otras distintas formas. El escritor traza en su universo literario sus propios espacios, tiempos y ensoñaciones, para dar rienda suelta a una historia. Las reflexiones de Genette comprenden un sincretismo entre la creación literaria y la intertextualidad, pero no únicamente en las conexiones de los propios textos literarios en sí, cuando aluden a contenidos, personajes o citas, sino como algo que va mucho más allá de lo escrito, que se nutre de historias o de ficciones presentes en otros formatos, tanto audiovisuales, quinestésicos, o de la misma experiencia del autor

\footnotetext{
${ }^{8}$ La "imitación", en este caso, implica el juego de dimensiones en el texto literario que se toman de nuestra realidad. Los personajes, espacios e ideas son una representación de las cuestiones filosóficas y existenciales del hombre en su contexto.
} 
(factores que, sumados a la iniciativa del investigador, terminan en la creación de una obra. De allí su variabilidad de influencias).

Obras como La máquina del tiempo (1895), de Herbert Wells, Ficciones (1944), de Jorge Luis Borges, Yo robot (1950), de Isaac Asimov, Otra vuelta de tuerca (1898), de Henry James, Frankestein (1818), de Mary Shelley, y El extraño caso del doctor Jekill y $\mathrm{Mr}$. Hide (1886), de Robert Stevenson, y Más allá del planeta silencioso (1938), de Clive Lewis, son maravillosas muestras de situaciones y personajes que difieren de los convencionalismos del mundo real, como los seres sobrenaturales o artificiales, espacios futurísticos, transformaciones humanas, dimensiones mezcladas y lugares inalcanzables. La fantasía en los textos literarios suele variar, bien sea por breves instantes, o por una permanencia contundente a lo largo de cada historia. Los eventos asombrosos adquieren una connotación gradual. De allí algunas de las propuestas de Tzvetan Todorov ${ }^{9}$, que reflejan la coexistencia (en la literatura) entre realidad y fantasía.

\section{Sombras de la creación: las grandes influencias del pasado}

Al momento de reflexionar sobre el proceso de creación literaria, Borges es uno de los escritores más interesantes. Su estilo es producto de una completa introspección, sumada a la potente filia por la lectura (especialmente por la gramática) y las conexiones que establece entre textos literarios cuyos contenidos están siempre en una interacción constante con factores y referentes del plano de la filosofía, la lingüística, la antropología y las ciencias exactas. Todo ello a través de un lenguaje literario que da cuenta de mundos relativos o divergentes con respecto al convencional. El tiempo y el espacio adquieren una connotación mutable; los lugares y épocas pueden ser reales o ficticios al mismo tiempo. Borges podría considerarse, en este sentido, un modelo ideal del creador literario, al asumir la literatura como una red en la cual los saberes, sin importar su índole, pueden mezclarse por medio de una historia, y porque sus textos generan en el lector la posibilidad de cuestionar e imaginar nuevas formas de percepción sobre la realidad.

El conocimiento de las ciencias exactas y naturales está regido, ciertamente, por unas leyes físicas y científicas que no pueden

\footnotetext{
9 Todorov (1980) realiza una reflexión sobre la literatura fantástica como género literario, e incluso propone algunos términos como "verosimilitud" y categorías de la ficción-no realista, entre ellas: "lo insólito", "lo fantástico" y “lo maravilloso".
} 
modificarse impunemente en la praxis del mundo objetivado. La realidad y el saber son el producto de una convención basada en la experiencia y en la percepción sensorial. Las lecturas borgianas permiten una mirada escéptica sobre ese mundo consensual, en la medida que todo puede pasar de todo en una historia fantástica, dada la presencia de hechos y acontecimientos divergentes del mundo objetivo. De esta forma, la "literatura fantástica clásica latinoamericana" y la "nueva literatura fantástica latinoamericana", denominada "ficción hipotética" ${ }^{10}$, proponen otras salidas y visiones de mundo en sus universos utópicos o distópicos, al mismo tiempo que tratan las problemáticas socioculturales en sus contextos y develan los comportamientos humanos.

Los lenguajes de Borges, Cortázar, Asimov, Lewis y Shelley son realmente llamativos, porque sus historias tratan de atmósferas y referentes que escapan de la realidad concreta, como también construyen personajes con determinadas actitudes e ideologías, y formulan, durante el desarrollo del texto, un conocimiento propio, que es la base de una obra de ciencia ficción. Los ya mencionados lenguajes de estos escritores no justifican la creación literaria bajo un ideal absoluto de causa y efecto, al decir que la puesta de sentido de una obra surge tras la lectura de los contenidos e imaginarios de un estado de arte. No se puede omitir la influencia y alusión de motivos o situaciones que van de una obra a otra, pero mucho más importante es entender que un texto tiene sus modos de tratar ciertos tópicos, en este caso, el fantástico, sea como algo remoto e inconcebible, o como algo cotidiano y permanente. Debe resaltarse no la conexión como hecho, sino más bien como un estado de arte que posee formas de enunciación y tópicos que le permiten al creador proponer su propio estilo, usando elementos ya trabajados. Actualizando una tradición literaria.

\section{Pasado del género: la dimensión paralela e invisible}

En Colombia la ciencia ficción puede considerarse escasa, debido al reducido número de textos y escritores consagrados a este género ${ }^{11}$.

\footnotetext{
${ }^{10}$ Para Croci (1990), la corriente narrativa denominada "nueva literatura fantástica latinoamericana" se origina en la síntesis entre la clásica literatura fantástica de nuestra América y lo que suele denominarse con la visión anglosajona de "ciencia ficción". Se habla de ficción hipotética o conjetural no porque sea una hipótesis o una conjetura el que exista tal tipo de ficción, sino porque se construye sobre el desarrollo narrativo de una premisa hipotética (premisa que puede ser de naturaleza científica, filosófica, o absolutamente imaginaria, fantástica).

${ }^{11}$ Burgos (1998) lleva a cabo una aproximación a la ciencia ficción colombiana, cuyas 
No hay una tradición consistente a la hora de hablar de Sci-fi colombiana. La problemática parte del pensamiento colectivo y hasta desinteresado (por no mencionar prejuicioso) de que la ciencia ficción y su producción, creadores y desarrollo, pertenecen únicamente al contexto anglosajón. Hay unas ideas sobre el género, siempre vistas hacia afuera, considerando su existencia bajo los imaginarios del primer mundo, o desde las producciones cinematográficas de Hollywood. Es necesario resaltar que "los relatos con acontecimientos irreales (ciencia-ficción, en este caso), responden a una necesidad de evasión de un mundo cotidiano que parece demasiado simple y desprovisto de sorpresas", una negación de la realidad, según Villate (2000), pues "la fantasía hace soñar con un mundo donde todo es posible y donde la tierra no es el único sitio en el que habita el ser humano [...] está el mundo sobrenatural, otras veces el cosmos o simplemente el devenir misterioso de la humanidad" (26). No debe haber mención de territorios, ni de culturas específicas para hablar de la validez o el reconocimiento de un género literario, porque la literatura construye mundos entre el hombre y la realidad, sin excepción de contextos.

A principios del siglo XX, con las ideas de modernidad y el progreso, Barranquilla se convirtió en el epicentro de las ideas modernas en Colombia, aun con una mayor independencia de la tradicional Atenas suramericana ${ }^{12}$. Desde allí comenzaron a consolidarse pequeños grupos que, en sus reuniones o tertulias, conversaban sobre temas como la prensa, la cultura, el arte, y por supuesto, la literatura ${ }^{13}$. Por otro lado, pese a su paradójica condición distópica, y

influencias se consolidaron desde el s XIX, con el contexto del pensamiento liberal y las ideas euro-pensantes sobre el progreso y la religión. Sin embargo, debido a la hegemonía del pensamiento conservador, a los regionalismos y reducidos grupos literarios -sumándose las fuertes corrientes literarias del siglo XX, como el realismo mágico y la literatura basada en la violencia colombiana-, la ciencia ficción ha sido un género invisibilizado, mantenido casi siempre dentro de las vanguardias.

${ }^{12}$ Bacca (1998) habla de Barranquilla como una ciudad con proyecciones burguesas que permitió, aun desde las reducidas élites, un espacio para la cultura. Se crearon revistas como Ideas (1920) y Lumen (1920), Caminos (1922), y en la década del cuarenta, La revista del museo, Mejoras y Civilización, y en la del cincuenta, Arte, Revista del Atlántico y Crónica, entre otras. En estas décadas, así mismo, el Grupo de Barranquilla estuvo integrado por varias personalidades como Gabriel García Márquez, José Félix Fuenmayor, Alejandro Obregón, Germán Vargas, Ramón Vinyes, Álvaro Cepeda Samudio. Lo más interesante, para este caso, es que en todos los debates y tertulias hubo un espacio para la ciencia ficción, desde Fuenmayor, cuyo relato Una triste aventura de catorce sabios (1928) abrió un nuevo campo, reducido y periférico, pero de profunda vitalidad.

${ }^{13}$ Comenzó entonces a construirse lentamente, no como eje central, sino como uno de los tantos temas, una propuesta (algo tenue) de lo que podría ser considerado como ciencia ficción colombiana. Entre los escritores que han trabajado en 
a los intereses de la crítica literaria colombiana, la ciencia ficción no es del todo desierta. Hay un sutil interés que ha permanecido hasta nuestros días, una estrecha trayectoria que ha persistido, a pesar de la invisibilización del género. Ya sea desde la academia, o a partir de la propia creación estética, la ciencia ficción colombiana sigue existiendo, aun a la espera de adquirir un reconocimiento como elemento constitutivo en la literatura nacional.

Si bien sobre la literatura del Caribe colombiano se han realizado estudios que implican acercamientos a problemáticas de exclusión social o racial, identidades de género, identidades sexuales, visiones de mundo desde el campo o lo urbano, violencia, denuncias sociales y testimonios, por mencionar algunos casos, hay muy pocos trabajos sobre ciencia ficción colombiana. Uno de los caminos debería ser preservar el interés por la ciencia ficción desde adentro, asumiéndola como una propuesta autónoma que pueda vincularse con factores identitarios y problemáticas del hombre, tanto a nivel regional como universal, y que continúe tratando de consolidar, como ya lo han hecho autores como Mora Vélez, Rebetéz y Burgos, un género literario que recurra a percepciones propias, antes que a determinismos anglosajones.

\section{El por qué de la ciencia ficción: la ecuación del texto}

Un género literario puede definirse con base a las consideraciones de la teoría, la historia y la crítica literaria, cuando diversos escritos (llámense cuentos, novelas, poemas, etc.) presentan en su conjunto características similares entre sí dentro de un plano espaciotemporal. Los elementos constitutivos de un texto, verbigracia: la narración, el tiempo, el espacio y las ideas, o focalizaciones sobre un determinado objeto o discurso, comparten constantes similares que responden ante los imaginarios y preocupaciones del ser humano en la sociedad. Un género literario es, además de una "taxonomía", el resultado de un conjunto de constantes que asocian un lenguaje literario con los hechos y los pensamientos del hombre.

A simple vista, la novela "parece un género homogéneo que se desenvuelve de manera exclusivamente autónoma durante siglos",

poca o gran medida este género, y que pueden considerarse clásicos, canónico o conocidos, encontramos a José Félix Fuenmayor, José Antonio Osorio Lizarazo, Manuel García Herreros, René Rebetéz, Germán espinosa y Antonio Mora Vélez, sumado a otros creadores más contemporáneos, como Orlando Mejía Rivera, Alberto Gaviria Coronado, Jesús Arango Cano, Rubén Ardila, Rafael de J. Henríquez, Jaime Restrepo Cuartas y Campo Burgos. 
como afirma Tinianov (1970). Pero en realidad, "no es un género constante sino variable y su material lingüístico, extra-literario, así como la manera de introducir ese material en literatura, cambian de un sistema literario a otro. Los rasgos del género evolucionan" (94). Con todas sus cualidades, la novela presenta "rasgos característicos" y "variaciones" de una época a otra. Las temáticas, el lenguaje literario, o los motivos, tienden a desaparecer, reforzarse o reaparecer tras varias décadas. La mutabilidad en la novela corresponde a cierto relativismo, al constante cambio de las ideologías en el hombre, y repercute en el texto literario. A esto llamaba Tinianov "evolución literaria" ${ }^{\prime 14}$.

El género de ciencia ficción, en el que podría inscribirse (si fuera necesario) una novela como Némesis, plantea unos imaginarios y temas donde el conocimiento, y sus diversas disciplinas, forman parte de un repertorio en el que se desarrollan las historias, que de por sí presentan hechos sobrenaturales o imprevisibles frente a nuestra realidad actual. No obstante, el hecho de trazar unas directrices propias no quiere decir que el género esté por fuera de una de las funciones de la literatura, que es la develación del ser humano y de diversos modos de conducta y pensamiento. En un texto de ciencia ficción hay, por ejemplo, tecnologías de vanguardia, aliens, naves espaciales y saberes con sus propias leyes (elementos tomados en poca o gran medida de nuestra realidad). Estos contenidos no son arbitrarios ni corresponden a un concepto cliché de la ciencia ficción, pues, al ser categorías de la otredad, implican un cuestionamiento a la evolución y las doctrinas del hombre. En la ciencia ficción también hay lugar para las emociones, los deseos y las ideologías que caracterizan a los seres humanos y que adquieren en el texto literario un carácter tan persistente como su propia episteme.

La literatura, y en especial la ciencia ficción, no solamente construye mundos sobrenaturales dominados por las más inusuales y excéntrica tecnologías. Es una cuestión que va mucho más allá del ingenio y la perspicacia de una "teoría científica". Este género construye universos. No teme a la refutación, o a las divergencias con otras disciplinas de nuestra materialidad, pues la obra de ciencia ficción estudia (desde la historia) su propio objeto y plantea (así como las novelas de otros tipos) una crítica a su género, una visión de mundo

\footnotetext{
${ }^{14}$ En Tinianov (1970 2004) el término "evolución literaria" no es un factor subjetivo que consiste en catalogar los escritos y sus cualidades bajo el grado de calificaciones positivas o negativas, sino que, más bien, trata de un seguimiento de las obras (especialmente desde la composición). Bajo esta aproximación literaria se pretende trasformar la literatura en una ciencia, al decir que esta presenta una trayectoria y unos fundamentos históricos que permitirán unos posibles objetos de estudio.
} 
estipulada desde su propia composición. Las obras literarias siempre estén desarrollando propuestas estéticas y temáticas particulares. Como afirmaba Kundera (2000), "la novela por sus propios medios, por su propia lógica, descubre los aspectos de la existencia”. La novela se convierte en una propuesta para con los lectores y críticos de un tiempo y un espacio, debido a que, en su red de significaciones, se desarrollan los devenires y cuestionamientos del hombre.

En la constitución del género novela, y en consecuencia de la ciencia ficción, hay dos factores primordiales al momento de configurar la obra: la "trama" y la "tensión". La trama indicará una sucesión que conlleva a los hechos desarrollados en la historia. Es el "hilo de Ariadna" con el cual el lector comprende la obra; un laberinto de posibilidades donde una acción conlleva a otra. La "tensión", por su parte, puede catalogarse como intriga. En la novela la tensión "no desaparece y el narrador consigue 'hechizar' a su audiencia con su canto. La noción moderna de intriga de acción se convierte en una intriga de predestinación, en la que la pregunta por lo que sucederá, es sustituida por lo mas sutil, sobre cómo se desarrollarán los hechos que ya sabemos que ocurrirán" (Todorov, 1976, citado por Atienza, 2003). La novela de ciencia ficción es bastante peculiar: además de mantener una trama y una tensión, debe sostener su propia "ciencia". Un planteamiento, un ideal o un dogma son factores develados en la historia, en el discurso, en la estética del lenguaje. La ciencia ficción soporta sus redes, argumenta con hechos (situaciones internas) las razones y la justificación de su propio conocimiento. El lector debe quedar seducido, atrapado, debe ser un cómplice mas en la red de eventos, sin ninguna otra opción que seguir el ritmo de la narración y hallar el cese, el descanso, la liberación de la intriga: un camino, un placer en la lectura de cada línea.

\section{Bibliografía}

Amoretti, M. (1992). Diccionario de términos asociados en teoría literaria. San José: Universidad de Costa Rica.

Béguin, A. (1997) Reacción y destino. Primer ensayo de crítica literaria. México: Fondo de Cultura Económica.

Bendezu, M. (2006). "Literatura fantástica”: http://www.monografias.com/ trabajos12/litfant/litfant.shtml?monosearch [Consultado el 20 de junio de 2010].

Bioy, A. (1965). "Prólogo a la Antología de la literatura fantástica": http://www.ciudadseva.com/textos/teoria/opin/bioy2.htm. [Consultado el 25 de octubre de 2010]. 
Bravo, V. (1996). Figuraciones del poder y la ironía. Caracas: Monte Ávila Editores.

Burgos, R. (1998). La ciencia ficción en Colombia. Tesis de Maestría. Bogotá, Universidad Javeriana.

Carreter, L. (1980). “Lengua literaria frente a lengua común”. En Estudios de lingüística. Barcelona: Crítica, pp. 149-171.

Croci, D. (1994). Literatura fantástica latinoamericana. Buenos Aires: E-ditores.

De Aguilar e Silva, V. M. (1972). Teoría de la literatura. Madrid: Gredos.

Eagleton, T. (1988). Una introducción a la teoría literaria. México: Fondo de Cultura Económica.

Fernández, C. (2008). Ángela viendo comer en McDonald's. Tesis de pregrado en creación literaria. Cartagena, Universidad de Cartagena.

Genette, G. (1993). Lenguaje poético, poética del lenguaje: http://es.scribd. com/doc/6805321/Gerard-Genette-Lenguaje-Poetico-PoeticaDel-Lenguaje [Consultado el 15 de agosto de 2011].

Gutiérrez, A. (2008). “Creación en narrativa, otra cara en investigación literaria", Visitas al Patio. Revista del Programa de Lingüística y Literatura, vol. I, $\mathrm{n}^{\circ} 1$, Cartagena, Universidad de Cartagena, pp. 75-87.

Gutiérrez Girardot, R. (1998). Jorge Luis Borges, el gusto de ser modesto. Siete ensayos de crítica literaria. Buenos aires: Panamericana

Huidobro, V. \& Lavarrete, L. (1989). Obra selecta. Caracas: Biblioteca Ayacucho.

Illán Bacca, R. (1998). Escribir en Barranquilla. Barranquilla: Uninorte.

Iser, W. (1987). El acto de leer: teoría del efecto estético. Madrid. Taurus.

James, F. \& Frager, R. (1976). Teorías de la personalidad. Buenos Aires: Alianza.

Kundera, M. (2000). El arte de la novela: http://losdependientes.com.ar/ uploads/1impyvasgf.pdf [Consultado el 10 de agosto de 2012].

Laverde, A. (2006). "Del canon a la tradición: el caso de la narrativa colombiana". En Los procesos de canonización de la novela colombiana en la historiografía literaria nacional. Medellín: Universidad de Antioquia.

Lotman, Y. (1978). “Composición de la obra artística verbal”. En Estructura del texto artístico. Madrid: Ediciones Istmo, pp. 261-342.

Melián, A. (1964). El romanticismo literario. Buenos Aires: Columba.

Rilke, R. M. (1999). Carta a un joven poeta. Barcelona: Edicomucación.

Said, E. (2004). El mundo, el texto y el crítico. Barcelona: Debate.

Sánchez, G. (2003). Creación, arte y psiquis. Bogotá: Academia Nacional de Medicina de Colombia.

Shergill, H. (2010). Psychology. New Delhi: Asoke K Ghosh.

Tinianov, J. (2004). “Sobre la evolución literaria”. En Todorov, T. (Comp.) Teoría de la literatura de los formalistas rusos. México: Siglo XXI.

Todorv, T. (1980). Introducción a la literatura fantástica. México: Premia. 
Villate, C. (2000). Realismo mágico latinoamericano: aproximaciones a su influencia en el periodismo de Héctor Rojas Herazo y Gabriel García Márquez. Tesis de pregrado. Universidad Pontificia Javeriana, Bogotá, Colombia. 